\title{
Reliability of non-invasive estimates of pulmonary hypertension by pulsed Doppler echocardiography
}

\author{
MITSUO MATSUDA, * TATSUHIKO SEKIGUCHI, YASURO SUGISHITA, \\ KENJI KUWAKO, KEIJI IIDA, IWAO ITO \\ From the Cardiovascular Division, Department of Internal Medicine, Institute of Clinical Medicine, \\ University of Tsukuba, Ibaraki, fapan
}

SUMMARY The duration of the acceleration phase of pulmonary systolic flow was measured by pulsed Doppler echocardiography in 39 normal subjects and 67 patients with heart disease to evaluate the reliability of this Doppler index as an estimate of pulmonary arterial pressure. The mean (SD) Doppler index in patients with abnormal mean pulmonary arterial pressure ( $>15 \mathrm{~mm} \mathrm{Hg}$ ) was significantly shorter than that in normal subjects $(110(30) \mathrm{ms}$ vs $150(10) \mathrm{ms}$ ). The Doppler index was significantly related to the mean pulmonary arterial pressure $(r=-0.75)$ the pulmonary blood flow $(r=0.46)$, and the total pulmonary vascular resistance $(r=-0.68)$. Forty four of 45 patients with an abnormal index ( $\leqslant 120 \mathrm{~ms}$ ) showed abnormal mean pressure $(>15 \mathrm{~mm} \mathrm{Hg})$. Without exception patients with a low index ( $\leqslant 90 \mathrm{~ms})$ had distinct pulmonary hypertension ( $\geqslant 25 \mathrm{~mm} \mathrm{Hg}$ ). Twelve of 22 patients with a normal index ( $\geqslant 130 \mathrm{~ms}$ ), however, also showed abnormal pressures. Nine of the 12 had an atrial septal defect and they had high pulmonary arterial pressure associated with high blood flow. Eighteen patients with valvar heart disease, whose mean pulmonary arterial pressure ranged from $16 \mathrm{~mm} \mathrm{Hg}$ to $24 \mathrm{~mm} \mathrm{Hg}$, had a significantly shorter acceleration phase and a higher total vascular resistance than 11 patients with atrial septal defect in whom the pressure range was similar $(120(20) \mathrm{ms}$ vs $140(20) \mathrm{ms}, 3 \cdot 8(1 \cdot 1)$ hybrid resistance unit $v s 1 \cdot 6(0.5)$ ).

Thus although the acceleration time of the pulmonary systolic flow is useful for the evaluation of pulmonary hypertension, it is a complex index that is affected not only by pulmonary arterial pressure but also by pulmonary blood flow and pathological changes in the pulmonary vascular bed.

Abnormal systolic flow patterns in the pulmonary artery and in the right ventricular outflow tract in patients with pulmonary hypertension have been detected in experimental studies by electromagnetic measurement of flow, ${ }^{1}$ and by clinical studies with Doppler echocardiography ${ }^{2-5}$ and contrast echocardiography. ${ }^{6}$ In pulmonary hypertension the pulmonary systolic flow begins to decelerate prematurely, ${ }^{2-5}$ with or without a subsequent re-acceleration in late systole. ${ }^{35}$ The initial acceleration phase of the systolic flow velocity curve in

Requests for reprints to Dr Mitsuo Matsuda, Institute of Health and Sport Sciences, University of Tsukuba, Sakura-mura, Niihari-gun, Ibaraki-ken 305, Japan.

*Present address: Institute of Health and Sport Sciences, University of Tsukuba, Ibaraki, Japan

Accepted for publication 12 March 1986 patients with pulmonary hypertension is characteristically shorter than that in normal subjects, ${ }^{4}$ and the duration of the acceleration phase decreases as the pulmonary hypertension progresses. ${ }^{45}$ This Doppler index may provide a non-invasive method for the evaluation of pulmonary arterial pressure. Pulmonary arterial pressure, however, depends on pulmonary flow volume and pulmonary vascular impedance. The duration of the acceleration phase in a patient with high flow volume may be different from that in another patient with high peripheral resistance, even if pulmonary arterial pressure is the same in both patients. Furthermore, right ventricular contractility may affect the pulmonary systolic flow pattern.

We have studied the relation between the duration of the acceleration phase of pulmonary systolic flow and haemodynamic variables in various diseases to 
evaluate the reliability of this Doppler index as an estimate of pulmonary arterial pressure.

\section{Patients and methods}

The study groups consisted of 39 control patients (17 men and 22 women; age range 20-48), 67 patients with heart disease ( 32 men and 35 women; age range 19-67) who underwent cardiac catheterisation, and three additional patients (two men and one woman; aged 28,62 , and 41 ) with severe right ventricular dysfunction (two had Uhl's anomaly and one had arrhythmogenic right ventricular dysplasia). Forty one of the 67 patients had valvar heart disease; 14 had an atrial septal defect, eight had ischaemic heart disease, three had primary pulmonary hypertension, and one had progressive systemic sclerosis.

Right heart catheterisation was performed within 1-6 days of pulsed Doppler echocardiographic investigation in the 70 patients with heart disease. Pulmonary arterial pressure (PAP) was measured by means of a fluid filled catheter and a pressure transducer (Electronics for Medicine). Pressure tracings were recorded on a recorder (Electronics for Medicine) at $50 \mathrm{~mm} / \mathrm{s}$ paper speed. Pulmonary blood flow volume (PBF) was obtained by the Fick method. Total pulmonary vascular resistance was calculated as $\mathrm{PBF} \div$ mean PAP. All patients were clinically stable, and none showed overt signs of congestive heart failure during the study.

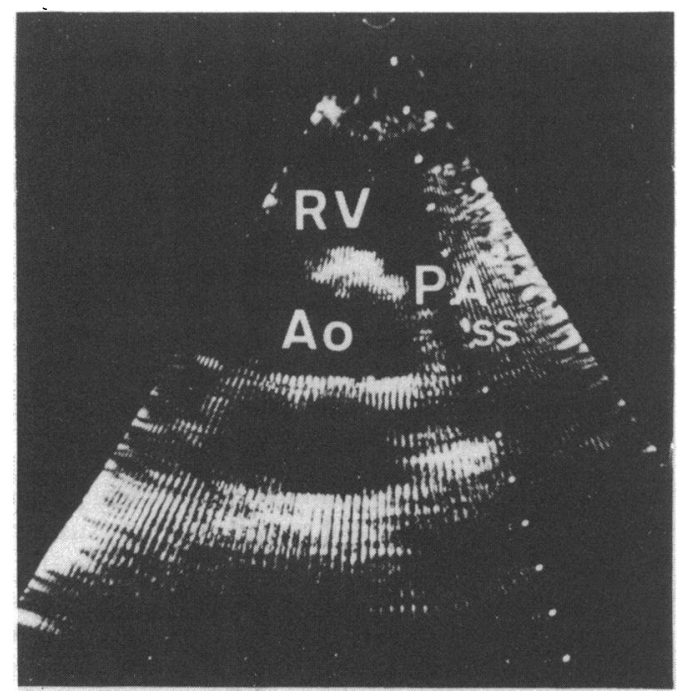

Fig 1 Sampling site of the Doppler signal. The pulmonary flow velocity curve was recorded at the root of the main pulmonary artery. $R V$, right ventricle; $P A$, pulmonary artery; Ao, aorta; ss, sampling site.
Pulsed Doppler echocardiograms were obtained with an ultrasonic pulsed Doppler unit combined with a cross sectional and an $M$ mode echocardiograph (Toshiba, or Aloka). The instruments have a $2.4 \mathrm{MHz}$ phased array transducer (Toshiba) and a $2.75 \mathrm{MHz}$ mechanical transducer (Aloka). After the sampling site of the Doppler signal had been established on the cross sectional image, the system was used in the Doppler mode (pulse repetition rates $6 \mathrm{KHz}$ (Toshiba) and $4.4 \mathrm{KHz}$ (Aloka)). Doppler output was obtained as spectral display by fast Fourier transformation. The bidirectional Doppler signal was recorded simultaneously with $M$ mode echocardiogram, phonocardiogram, and electrocardiogram on a strip chart recorder (Honeywell) at a $50 \mathrm{~mm} / \mathrm{s}$ paper speed. The examinations were performed with the patient supine and the transducer on the parasternal area. The sampling site for Doppler echocardiography was positioned distal to the pulmonary valve leaflets in the main pulmonary artery (fig 1). The width of the sampling volume along the ultrasonic beam was $2 \mathrm{~mm}$. Kitabatake et al preferred to measure the flow in the right ventricular outflow tract rather than the pulmonary arterial flow, because the Doppler signals from the flow obtained in the latter position were often affected by a mixing of signals caused by systolic fluttering of the pulmonary valve cusp, by eddy currents in patients with pulmonary hypertension, or by both. ${ }^{5}$ We, however, were able to record flow velocity curves more clearly in the pulmonary artery than in the right ventricular outflow tract, when the sampling site was located in the middle of the pulmonary trunk distal to the pulmonary valve.

Doppler velocity flow curves showed that systolic ejection flow was away from the transducer, that is

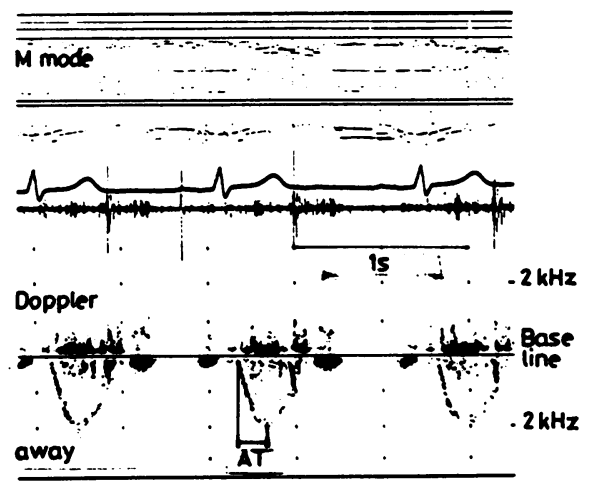

Fig 2 Pulmonary flow velocity curve in a normal subject. The course of ejection flow velocity is shown as a monophasic curve peaking in mid-systole and sustained to end systole. The acceleration time ( $A T$ ) was measured from the onset of ejection to the peak flow velocity. 


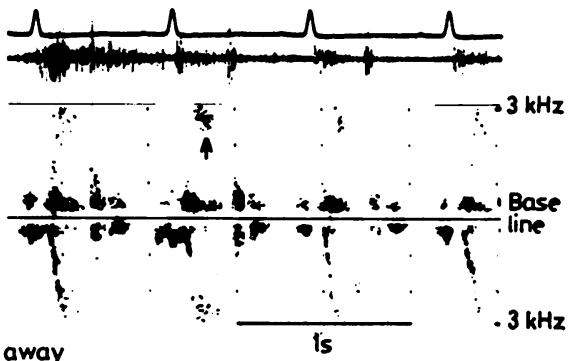

Fig 3 Flow velocity curve in a patient with high flow velocity. When flow velocity exceeded the maximum detectable velocity of the pulsed Doppler method, the aliased Doppler signal (arrow) is displayed on the opposite side of the baseline. The time of the peak flow was determined from this aliased signal.

from the right ventricular outflow tract into the main pulmonary artery (fig 2). The duration of the acceleration phase of ejection flow (acceleration time) was measured from the onset of ejection to the peak flow velocity, as shown in fig 2 . Acceleration time was averaged over ten consecutive cardiac cycles. When flow velocity exceeded the maximum detectable velocity, which depends on the ultrasonic pulse repetition rate, the aliased Doppler signal was displayed above the base line as shown in fig 3 . In most of these cases the time of peak flow could be determined from the aliased signal. In seven patients with atrial septal defect, however, the peak flow could not be measured exactly. In these patients we used the flow velocity curve of the right ventricular outflow tract to measure acceleration time. The difference between the acceleration time value at the pulmonary artery and that at the right ventricular outflow tract was calculated by comparison of these values in 14 patients (nine patients with valvar heart disease and five with atrial septal defect).

Kitabatake et al used the ratio of acceleration time to right ventricular ejection time to evaluate pulmonary hypertension. ${ }^{5}$ We, however, were not always able to determine exactly the end point of right ventricular ejection if the late systolic part of the flow curve showed a stationary or backward flow pattern. Moreover, there was more beat to beat variation in ejection time than in acceleration time, especially in patients with atrial fibrillation (fig 4b). Thus we did not use right ventricular ejection time.

Data were analysed by the $t$ test for comparison of means between the two groups. Relations between variables were analysed by linear regression. The significance of difference between correlation coefficients was tested by Fisher's $Z$ transformation of the coefficients. The level of significance was set at $\mathrm{p}<0.05$. (a)

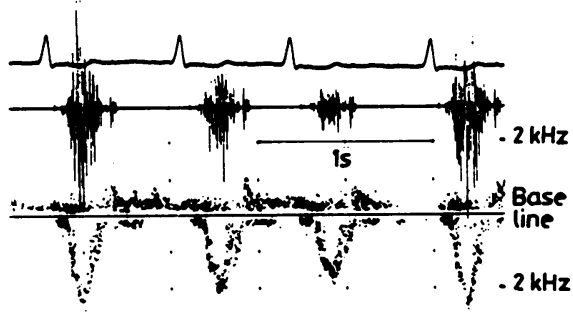

(b) away
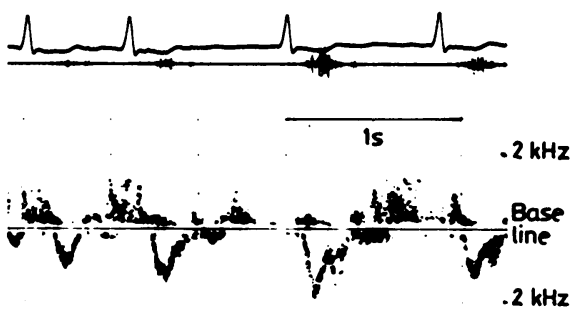

away

(c)
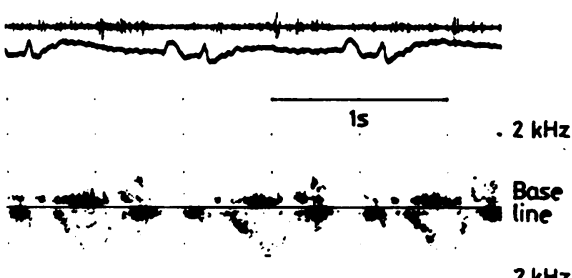

away

Fig 4 Flow velocity curves in patients with various heart diseases. (a) In pulmonary hypertension the peak of the flow appeared prematurely and was followed by a sharp fall in flow velocity. In atrial fibrillation (b) the late systolic part of the flow curve changed from beat to beat. There was re-acceleration of flow in the third and the fourth cycles. In the first beat there was no flow or reversed flow in the right ventricular outflow tract. In a patient with a weakly contracting right ventricle (c) the course of the flow curve resembled the normal flow pattern; however, the velocity reached a peak in late systole.

\section{Results}

The systolic flow velocity curve in normal patients started at the beginning of systole and was sustained to the end of systole, peaking in mid-systole (fig 2). In most patients with pulmonary hypertension, the peak of the flow velocity appeared prematurely, and there was a sharp decrease in flow velocity in midsystole (fig 4a). The later part of the systolic curves showed two characteristic kinds of pattern, a reaccelerated flow that produced a second peak and a neutral or sometimes reversed flow (fig 4b). One of 
Table 1 Acceleration time (mean (SD)) measured in the pulmonary artery and in the right ventricular outflow tract

\begin{tabular}{lllll}
\hline & $\begin{array}{l}\text { Patient } \\
n o\end{array}$ & \multicolumn{2}{l}{$A T(m s)$} & \\
\cline { 3 - 4 } & & $P A$ & RVOFT & $p$ \\
\hline VHD & 9 & $110(20)$ & $110(20)$ & NS \\
ASD & 5 & $130(40)$ & $140(40)$ & NS \\
\hline
\end{tabular}

AT, acceleration time of pulmonary ejection flow; PA, pulmonary artery; RVOFT, right ventricular outflow tract; VHD, valvar heart disease; ASD, atrial septal defect.

the patterns was observed for individual patients or for different beats in patients with atrial fibrillation. The course of the ejection flow curve in patients with impaired contraction of the right ventricle resembled the normal flow pattern, though the flow velocity peaked in late systole (fig 4c).

There were no significant differences between the acceleration time determined at the pulmonary artery and that determined at the right ventricular outflow tract (table 1). The mean (SD) acceleration time in control subjects was $150(10) \mathrm{ms}$ with a range

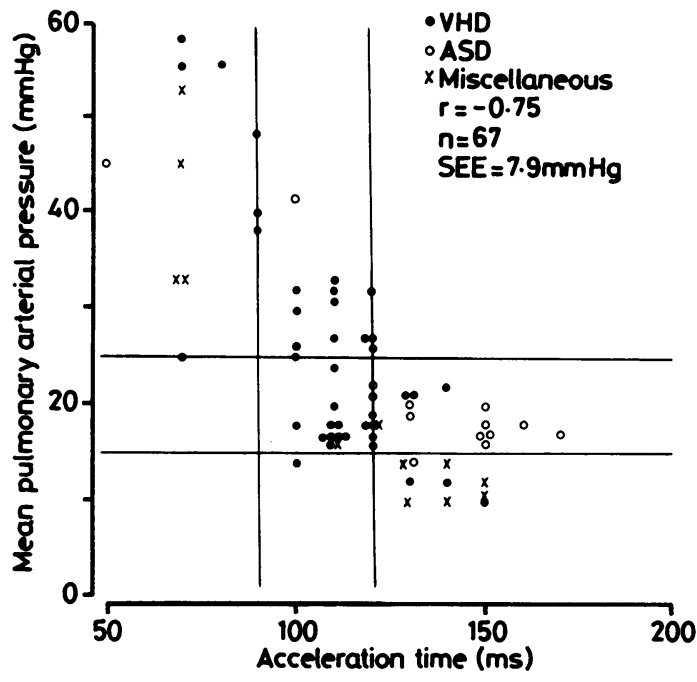

Fig 5 Relation between acceleration time $(A T)$ and mean pulmonary arterial pressure ( $M P A P)$ in patients with various heart diseases. The acceleration time decreased with an increase in pulmonary arterial pressure. The linear regression equation was $m P A P=65-0.35 A T$ and the $F$ value was $86(F(0.05 \%)=3.9)$. Ten of 11 patients with normal pressure ( $<15 \mathrm{~mm} \mathrm{Hg}$ ) showed a normal $A T$ $(\geqslant 130 \mathrm{~ms}$ ). All 25 patients with mean pressure $>25 \mathrm{~mm} \mathrm{Hg}$ had an abnormal value ( $<120$ ms). Patients with an extremely short $A T$ ( $\leqslant 90 \mathrm{~ms}$ ) had distinct pulmonary hypertension ( $\geqslant 25 \mathrm{~mm} \mathrm{Hg}$ ). Twelve of 22 patients with a normal $A T$, however, had abnormal pulmonary artery pressure. Nine of these 12 subjects had atrial septal defect ( $A S D)$. VHD, valvar heart disease.

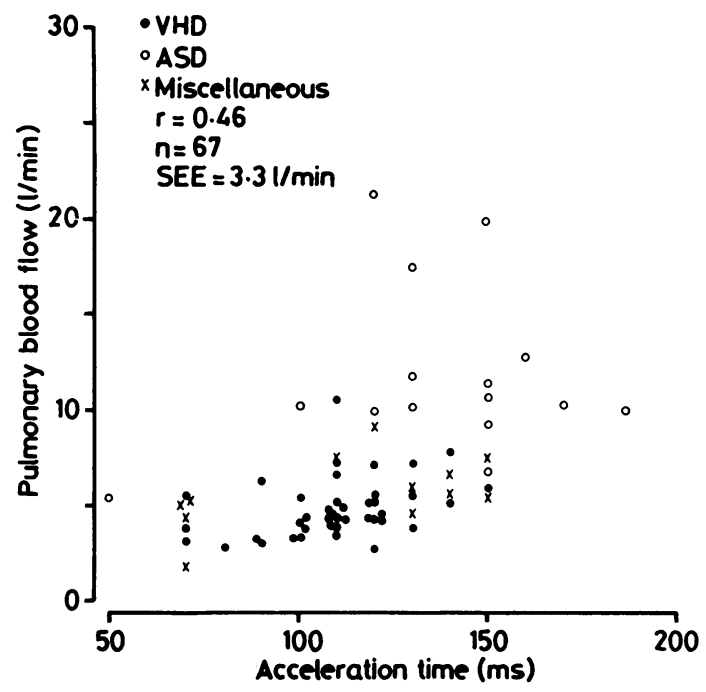

Fig 6 Relation between acceleration time $(A T)$ and pulmonary blood flow ( $P B F)$. The linear regression equation was $P B F=-1.4+0.069 A T$ and the $F$ value was 18 . Most patients with atrial septal defect ( $A S D$ ) had a long $A T$ and high blood flow volume. VHD, valvar heart disease.

from $130 \mathrm{~ms}$ to $180 \mathrm{~ms}$. The acceleration time in those with heart disease ranged from $50 \mathrm{~ms}$ to $170 \mathrm{~ms}$ $(110(30) \mathrm{ms})$, and was inversely related to mean pulmonary arterial pressure $(r=-0.75, \mathrm{p}<0.001$ (fig 5)).

The acceleration time was significantly shorter in patients with an abnormal mean pulmonary arterial pressure $\left(>15 \mathrm{~mm} \mathrm{Hg}^{7}\right)$ than in patients without heart disease $(110(30) \mathrm{ms}, \mathrm{p}<0.001)$. When abnormal acceleration time was defined as being $<120 \mathrm{~ms}$ (according to the range of the values in the controls), 44 of 45 patients with an abnormal index had an abnormal mean pulmonary arterial pressure. All 25 patients whose mean pulmonary arterial pressure was $>25 \mathrm{~mm} \mathrm{Hg}$ had an abnormal acceleration time. All 12 patients in whom acceleration time was $<90 \mathrm{~ms}$ had definite pulmonary hypertension (mean pulmonary arterial pressure $\geqslant 25 \mathrm{~mm} \mathrm{Hg}$ ). Ten of 11 patients with a normal pulmonary arterial pressure had a normal acceleration time. Twelve of 31 patients, however, whose mean pulmonary arterial pressure ranged from $16 \mathrm{~mm} \mathrm{Hg}$ to $24 \mathrm{~mm} \mathrm{Hg}$ had a normal acceleration time. Twelve of the 22 patients with normal acceleration time ( $\geqslant 130 \mathrm{~ms})$ showed abnormal mean pulmonary arterial pressure $(>15 \mathrm{~mm} \mathrm{Hg})$. Nine of these 12 subjects had atrial septal defect.

Acceleration time also correlated with the pulmonary blood flow volume $(r=0.46, p<0.001$ (fig 6)), and the total pulmonary vascular resistance 
162

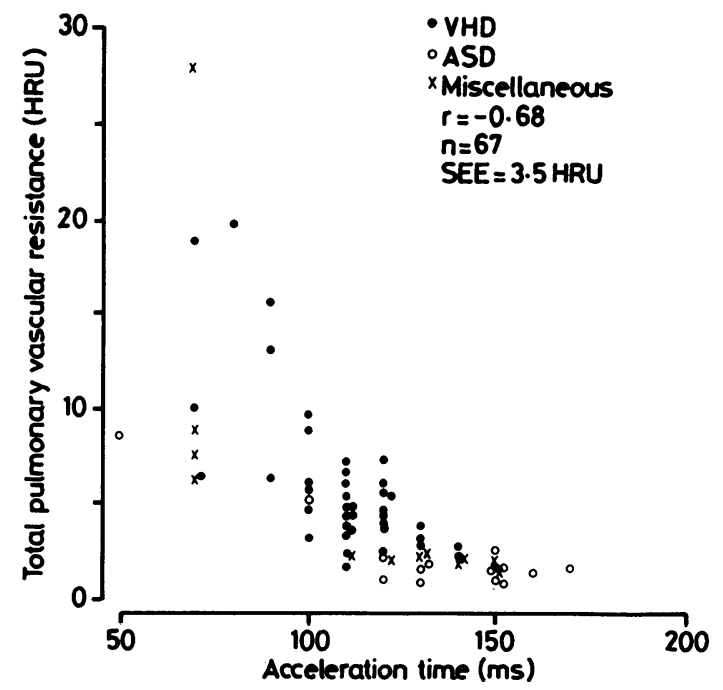

Fig 7 Relation between acceleration time ( $A T)$ and total pulmonary vascular resistance $(T P R)$. The linear regression equation was $T P R=2 \cdot 0-0 \cdot 13 A T$ and the $F$ value was 57. Patients with atrial septal defect ( $A S D)$ who had long acceleration time had low vascular resistance. VHD, valvar heart disease.

$(\mathrm{r}=-0.68, \mathrm{p}<<0.001$ (fig 7)). The correlation between acceleration time and pulmonary blood flow volume was not as strong as that between acceleration time and mean pulmonary arterial pressure $(p<0.001)$. Patients with atrial septal defect, whose pulmonary blood flow volume was generally high, tended to have longer acceleration times (fig 6). The correlation coefficient between acceleration time and total pulmonary vascular resistance was not significantly different from that between acceleration time and mean pulmonary arterial pressure. The total pulmonary vascular resistance in patients with atrial septal defect with a normal acceleration time, however, tended to be low (fig 7).

Relations between acceleration time and haemodynamic function appeared to be curvilinear (fig 5 and fig 7). When acceleration time was compared with $\log _{10}$ mean pulmonary arterial pressure (mPAP) and $\log _{10}$ total pulmonary vascular resistance (TPR), the correlation coefficients were
Matsuda, Sekiguchi, Sugishita, Kuwako, Iida, Ito -0.75 and -0.79 respectively. Again there was no significant difference between these correlation coefficients. The regression equations for these relations were $\log _{10} \quad(\mathrm{mPAP})=2 \cdot 0-0.0057 \mathrm{AT}$ $(\mathrm{F}$ value $=83, \mathrm{SEE}=0.13)$, and $\log _{10}(\mathrm{TPR})=$ $1.8-0.010 \mathrm{AT} \quad(\mathrm{F}$ value $=108, \quad \mathrm{SEE}=0.21)$ respectively, where AT is acceleration time and SEE is standard error of estimate.

The acceleration time in 11 patients with atrial septal defect in whom mean pulmonary arterial pressure ranged from $16 \mathrm{~mm} \mathrm{Hg}$ to $24 \mathrm{~mm} \mathrm{Hg}$ was significantly longer than that in 18 patients with valve disease in whom the range of mean pulmonary arterial pressures was similar $(19(2) \mathrm{mm} \mathrm{Hg}$ vs 19 (2) $\mathrm{mm} \mathrm{Hg}, \mathrm{NS}$; acceleration time $(140(20) \mathrm{ms}$ vs $120(10) \mathrm{ms}, \mathrm{p}<0.005)$. The same patients with atrial septal defect had significantly lower total pulmonary vascular resistance than patients with valve disease $(1.6(0.5)$ HRU vs $3.8(1.1)$ HRU, $p<0.001)$. Patients with atrial septal defect in whom total pulmonary vascular resistance was increased and in whom pulmonary hypertension was severe had an abnormal acceleration time, however (fig 5 and fig 7). The correlation coefficient between acceleration time and mean pulmonary arterial pressure in patients with atrial septal defect was -0.87 ; in the remainder it was -0.78 . The regression equation for acceleration time (AT) in patients with atrial septal defect was $\mathrm{mPAP}=57-0 \cdot 27 \mathrm{AT}(\mathrm{F}$ value $=$ 36 , standard error of estimate $=4.8 \mathrm{~mm} \mathrm{Hg}$ ) and that in the other patients was mPAP $=74-0.44 \mathrm{AT}$ $(F$ value $=78, S E E=8.0 \mathrm{~mm} \mathrm{Hg})$.

In the three patients with severe right ventricular dysfunction the acceleration time was longer than normal ( $160-230 \mathrm{~ms}$, table 2$)$. We did not apply statistical tests to this small group. Right ventricular angiography showed a dilated right ventricle with poor systolic function and tricuspid regurgitation. There was a slight increase in mean pulmonary arterial pressure and total pulmonary vascular resistance, which we think is caused by a rise in pulmonary capillary pressure (table 2 ).

\section{Discussion}

The acceleration time of pulmonary arterial systolic

Table 2 Acceleration time and haemodynamic data in patients with severe right ventricular dysfunction

\begin{tabular}{|c|c|c|c|c|c|}
\hline Patient No & $A T$ (ms) & $\begin{array}{l}\text { Mean } P A P \\
(\min H g)\end{array}$ & $P B F$ (l/min) & $T P R(H R U)$ & $\begin{array}{c}\text { Mean } P C \\
(\operatorname{mm} H g)\end{array}$ \\
\hline $\begin{array}{l}1 \\
2 \\
3\end{array}$ & $\begin{array}{l}160 \\
230 \\
230\end{array}$ & $\begin{array}{l}17 \\
14 \\
16\end{array}$ & $\begin{array}{l}4.5 \\
3.9 \\
-\end{array}$ & $\begin{array}{l}3.8 \\
3.6 \\
-\end{array}$ & $\frac{15}{12}$ \\
\hline
\end{tabular}

AT, acceleration time; mean PAP, mean pulmonary arteriat presstre, PBF; puilmotiary blood flow; TPR, total peripheral vascular resistance; mean PC, mean pulmonary capillary pressure. 
flow, measured by Doppler echocardiography, decreased with an increase in pulmonary arterial pressure. The correlation coefficient obtained in this study $(r=-0.75)$ resembled that reported by Kitabatake et al $(\mathrm{r}=-0.82) .^{5}$ Most patients with an abnormal index had abnormal pulmonary arterial pressure. Without exception, patients with an extremely short acceleration time $(\leqslant 90 \mathrm{~ms})$ had prominent pulmonary hypertension. In all patients with pulmonary hypertension the acceleration time was abnormal, and in most patients with normal pulmonary arterial pressure it was normal. Thus echocardiographic measurement of the acceleration phase of pulmonary arterial ejection flow at first appeared to be a promising non-invasive index of pulmonary hypertension.

In 12 of 22 patients with a normal index, however, pulmonary arterial pressure was abnormal (that is the mean pressure was $>15 \mathrm{~mm} \mathrm{Hg}$ and $<25 \mathrm{~mm} \mathrm{Hg}$ ). Nine of these 12 patients had an atrial septal defect. In this study pulmonary blood flow was high in most patients with atrial septal defect. The acceleration time of ejection flow correlated not only with pulmonary arterial pressure, but also with pulmonary blood flow volume, that is the acceleration time increased with an increase in blood flow volume. Patients with an atrial septal defect and a normal Doppler index tended to have high pulmonary pressures associated with high blood flow volumes. Pulmonary vascular resistance was low in these patients. In contrast, the acceleration time was shorter than normal in patients with valvar heart disease, who had comparable pulmonary arterial pressure and higher peripheral resistance. The Doppler index was abnormal even in patients with atrial septal defect when pulmonary vascular resistance was increased. These results suggest that the duration of the acceleration phase of pulmonary systolic flow is more strongly influenced by pathological changes in the pulmonary vascular bed than by pulmonary arterial pressure itself.

The premature deceleration of pulmonary systolic flow in patients with pulmonary hypertension indicates that the pressure gradient between the right ventricular outflow tract and the pulmonary artery is reversed and counteracts systolic flow earlier than usual. In an earlier study we proposed two mechanisms to explain this early reversal of the pressure gradient in patients with pulmonary hypertension ${ }^{3}$ : that decreased distensibility of the vessel wall ${ }^{8}$ may increase the amplitude of the pressure wave propagated from the right ventricular outflow tract into the pulmonary trunk; and that the reflection of the pressure wave at the peripheral vascular bed is increased by reduced vessel calibre or obstruction of the pulmonary arterioles and capillaries, so that the reflected wave front reaches the pulmonary trunk prematurely through the stiffened vessels in such patients. These two mechanisms would result in an earlier reversal of the pressure gradient, that in turn would produce earlier deceleration of pulmonary systolic flow.

Our results are compatible with the finding reported by Milnor et al that pressure wave velocity was highest in the subjects with the highest pulmonary vascular resistance at any given mean pressure. ${ }^{9}$ They suggested that the increases in pulmonary arterial pulse wave velocity and impedance in their patients were not entirely due to passive distension of the artery by high transmural pressure, and that the pathological stiffening of the larger arteries in patients with high vascular resistance was greater than that produced by an uncomplicated increase in distending pressure. Patients with high pulmonary vascular resistance are likely to have histological alterations both in the peripheral vascular bed and in the large arteries; these will cause higher pressure peaks, a higher flow velocity, and a larger reflection of the pressure pulse wave. All of these effects will result in an earlier deceleration of pulmonary systolic flow than in patients with low pulmonary vascular resistance.

The acceleration phase of pulmonary systolic flow was prolonged in three patients with severe right ventricular dysfunction. Mean pulmonary arterial pressure and total peripheral vascular resistance were slightly increased. The increase may have resulted from raised pulmonary venous pressure rather than histological changes in the pulmonary arteries. Impaired ventricular contraction alone might not cause an early deceleration of pulmonary systolic flow if the pulmonary vascular bed is normal. In these three patients right ventricular angiography showed pronounced tricuspid regurgitation. Kitabatake et al reported that the good correlation between the Doppler acceleration index and mean pulmonary arterial pressure held irrespective of underlying tricuspid regurgitation; however, the acceleration time tended to be short in patients with mild or moderate tricuspid regurgitation. ${ }^{5}$ This may indicate that the Doppler acceleration index is not strongly affected by tricuspid regurgitation.

Although the acceleration time of pulmonary systolic flow is a useful index for the evaluation of pulmonary hypertension, it is affected by pulmonary arterial pressure, pulmonary blood flow, and histological changes in the pulmonary vascular bed. The acceleration time is a specific but not a sensitive index of pulmonary arterial pressure. An abnormal index indicates raised blood pressure with high peripheral resistance, but a normal index does not exclude raised blood pressure associated with high 
164

blood flow. Separate evaluation of each disease group should, however, improve the accuracy of this index. The correlation coefficient between acceleration time and mean pulmonary arterial pressure in patients with atrial septal defect was -0.87 (SEE $4.8 \mathrm{~mm} \mathrm{Hg}$ ), and that in the remainder of patients was -0.78 (SEE $8.0 \mathrm{~mm} \mathrm{Hg}$ ). This Doppler index is also suitable for the assessment of the pulmonary vasular bed.

We thank Miss Yoko Kojima for technical assistance.

\section{References}

1 Tahara M, Tanaka H, Nakao S, et al. Hemodynamic determinations of pulmonary valve motion during systole in experimental pulmonary hypertension. Circulation 1981;64:1249-55.

2 Sakakibara H, Miyatake K, Kinoshita N, et al. Noninvasive detection of shunt flow in atrial septal defect with a compound of the ultrasonic pulsed Doppler technique and cross-sectional echocardiography. f Cardiogr 1980;10:225-34.

3 Matsuda M, Sugishita Y, Yamaguchi T, Tamura T, Ito
Matsuda, Sekiguchi, Sugishita, Kuwako, Iida, Ito

I. Pulmonary flow velocity patterns in patients with pulmonary hypertension: a study using pulsed Doppler echocardiography combined with twodimensional echocardiography. $\mathcal{f}$ Cardiogr 1981;11: 603-14.

4 Matsuda M, Sugishita T, Yamaguchi T, et al. Pulmonary flow velocity pattern in patients with pulmonary hypertension: a study using pulsed Doppler echocardiography [Abstract]. fpn Circ $f$ 1981;45:921.

5 Kitabatake A, Inoue $M$, Asao $M$, et al. Noninvasive evaluation of pulmonary hypertension by a pulsed Doppler technique. Circulation 1983;68:302-9.

6 Meltzer RS, Valk NK, Visser CA, Roelandt J. Contrast echocardiography in pulmonary hypertension: observations explaining the early closure of the pulmonic valve. Am Heart f 1983;196:1394-8.

7 The criteria committee of the New York Heart Association. Nomenclature and criteria for diagnosis of diseases of the heart and great vessels. 7th ed. Boston: Little, Brown, 1973:276-7.

8 Greenfield JC Jr, Griggs DM Jr. Relation between pressure and diameter in main pulmonary artery of man. I Appl Physiol 1963;18:557-9.

9 Milnor WR, Conti CR, Lewis KB, O'Rourke MF. Pulmonary arterial pulse wave velocity and impedance in man. Circ Res 1969;25:637-49. 